\title{
Doenças pós-colheita em laranja 'Valência'e caracterização da população fúngica em pomares orgânicos e convencionais
}

\author{
Ivan H. Fischer ${ }^{1}$, Micheli M. Zanette ${ }^{1}$, Marcel B. Spósito² \& Lilian Amorim³ \\ 'Laboratório de Fitossanidade, APTA Centro Oeste, 17030-000, Bauru, SP, Brasil; ${ }^{2}$ Departamento de Produção Vegetal, \\ Universidade de São Paulo, ESALQ, 13418-900, Piracicaba, SP, Brasil; ${ }^{3}$ Departamento de Fitopatologia e Nematologia, \\ Universidade de São Paulo, ESALQ, 13418-900, Piracicaba, SP, Brasil
}

Autor para correspondência: Ivan H. Fischer, e-mail: ihfische@apta.sp.gov.br

\begin{abstract}
RESUMO
A agricultura orgânica busca atender a demanda crescente por alimentos saudáveis, produzidos sem agroquímicos. Este trabalho objetivou: a) caracterizar as doenças pós-colheita em laranjas 'Valência' provenientes de cultivo orgânico e convencional; b) caracterizar a micoflora ambiental nos pomares citrícolas e c) detectar a presença de isolados de Penicillium spp. resistentes aos fungicidas tiabendazol e imazalil nos pomares. Frutos coletados nos pomares e no mercado atacadista de São Paulo (CEAGESP) foram armazenados durante 14 dias a $25^{\circ} \mathrm{C}$ e $85 \%$ de UR. A incidência de doenças foi avaliada visualmente a cada dois-três dias. A micoflora ambiental foi amostrada com placas de Petri, contendo meio batata-dextrose-ágar acrescido ou não dos fungicidas. As maiores incidências de doenças foram observadas nas laranjas orgânicas em relação as do sistema convencional. A principal doença detectada foi a podridão peduncular de lasiodiplodia. A micoflora total do ambiente, assim como os principais gêneros fúngicos não diferiram significativamente entre os pomares amostrados. Cladosporium foi o gênero fúngico mais abundante nos pomares. A frequência de isolados de $P$. digitatum resistentes a tiabendazol foi semelhante nos pomares, com média de $47,3 \%$ dos isolados. A frequência de isolados de $P$. digitatum resistentes a imazalil foi baixa $(2,6 \%)$, não sendo constatados isolados resistentes a tiabendazol + imazalil.
\end{abstract}

Palavras-chave: Citrus sinensis, Penicillium spp., citros, podridões, resistência a fungicidas.

\begin{abstract}
Postharvest diseases in orange fruits and characterization of the fungal population in organic and conventional farmers

Organic farming aims to meet growing demand for healthy foods, produced without the use of pesticides. The purposes of this work were a) to characterize postharvest diseases in 'Valencia' oranges grown under organic and conventional conditions; b) to characterize the environmental mycoflora in citrus orchards and c) to detect the presence of Penicillium spp. isolates resistant to fungicides thiabendazole and imazalil in the orchards. Citrus fruit collected at the orchards and at the wholesale market of São Paulo (CEAGESP) were stored during 14 days at $25^{\circ} \mathrm{C}$ and $85 \%$ UR. The incidence of postharvest diseases was evaluated visually every two-three days. The environmental mycoflora was sampled according to the gravimetric method, using Petri dishes containing potato-dextrose-agar medium, supplemented or not with fungicides. The highest incidence of diseases was observed in oranges sampled in organic orchards in comparison to the conventional system. The most frequent postharvest disease was lasiodiplodia stem-end rot. The environmental mycoflora and the population of the major genera of fungi did not differ significantly among the sampled orchards. Cladosporium was the most prevailing genus in the orchards. The frequency of $P$. digitatum isolates resistant to thiabendazole was similar in orchards, with an average of $47.3 \%$. The frequency of $P$. digitatum isolates resistant to imazalil was low (2.6\%), and resistance to thiabendazole + imazalil was not observed. Key words: Penicillium spp., citrus, fungicide resistance, rots.
\end{abstract}

\section{INTRODUÇÃO}

As técnicas de manuseio e conservação pós-colheita de frutos vêm recebendo grande atenção nos últimos anos, pois os danos nesta fase podem superar $20 \%$, decorrentes principalmente de doenças (Dantas et al., 2003; Fischer et al., 2008). Em frutos cítricos coletados diretamente de packinghouses paulistas, a incidência de doenças foi superior a 14\% em laranjas (Citrus sinensis L Osbeck) e em tangor 'Murcott' (C. sisnesis x C. reticulata Blanco), após duas semanas de armazenamento a $25^{\circ} \mathrm{C}$ (Fischer et al., 2007). A doença mais frequente naquele levantamento foi o bolor verde, causado por Penicillium digitatum (Pers.:Fr.)
Sacc. Entretanto, em frutos destinados à exportação, a incidência ficou abaixo de $3 \%$, com predomínio da podridão peduncular, causada por Lasiodiplodia theobromae (Pat.) Griffon \& Maubl. em laranja 'Valência'. Evidenciou-se que as boas práticas agrícolas adotadas nos packinghouses de exportação, que incluem seleção de frutos com baixa fonte de inóculo dos patógenos, eficiência do processo de seleção de frutos injuriados e tratamento com $\mathrm{NaClO}$ e fungicidas em pós-colheita são importantes para o controle de doenças pós-colheita (Fischer et al., 2009b).

Os índices de descarte de frutos em pós-colheita são geralmente reflexos da incidência de doenças nessa fase, uma vez que os frutos são desqualificados para comercialização 
pela presença dos sintomas (Gullino, 1994). A probabilidade de infecção na pós-colheita depende da quantidade de inóculo presente em um ponto do fruto suscetível. Esta relação com frutos cítricos foi demonstrada para patógenos importantes, como P. digitatum (Wild \& Eckert, 1982) e Colletotrichum gloeosporioides (Penz.) Penz. et Sacc. (Brown, 1975), segundo a qual, a quantidade de esporos presentes nos packinghouses influiu significativamente nos níveis de doenças pós-colheita (Tuset, 1987; Eckert \& Eaks, 1989; Zhu et al., 2006). Em levantamento da micoflora em packinghouses de citros paulistas foi constatado predomínio dos gêneros de fungos Cladosporium e Penicillium (Fischer et al., 2009a). Entretanto, entre os patógenos de pós-colheita com importância econômica em citros, os gêneros Penicillium e Alternaria foram os isolados com maior frequência nestes packinghouses (Fischer et al., 2009a), assim como em pomares cítricos espanhóis (Palou et al., 2001a).

Os problemas derivados dos altos níveis de população fúngica nos pomares e packinghouses de citros são significativamente aumentados quando existem isolados dos patógenos resistentes aos fungicidas empregados (Fischer et al., 2009a). Estudos desenvolvidos, principalmente a partir de 1970, têm constatado que o fenômeno de resistência é uma das principais causas do fracasso de muitos tratamentos com fungicidas em pós-colheita (Houck, 1977; Boubaker et al., 2009). A grande maioria dos estudos se refere, por sua importância econômica, a $P$. digitatum e $P$. italicum Wehmer frente aos fungicidas do grupo dos benzimidazóis, ortofenilfenato de sódio e imazalil, e descrevem casos, tanto de resistência simples, como de resistência cruzada e múltipla (Bus et al., 1991; Schmidt et al., 2006; Zhu et al., 2006; Boubaker et al., 2009). Amostragens do ambiente de packinghouses de citros paulistas constataram que 39\% dos isolados de $P$. digitatum apresentavam resistência ao fungicida tiabendazol e $1 \%$ ao imazalil (Fischer et al., 2009a). A presença de $P$. digitatum resistente a fungicidas em elevada frequência nos packinghouses sugere o campo como o principal local para ocorrência de resistência, provavelmente pelo alto número de aplicações de fungicidas feitas durante quase todo ciclo de produção, no controle de diferentes doenças. Isso ficou evidente, pois a maior frequência de isolados resistentes foi encontrada em packinghouses que não empregava fungicidas pós-colheita (Fischer et al., 2009a).

À medida que se questiona a aplicação de fungicidas sintéticos para o controle de doenças, por seus possíveis efeitos nocivos à saúde e ao meio ambiente, vem crescendo o interesse por sistemas de cultivo agroecológico, como o orgânico, com um grande nicho de mercado a ser explorado. O sistema de cultivo orgânico de citros vem sendo adotado em vários estados brasileiros (Camargo et al., 2006). Entretanto, o principal entrave para a produção de laranja orgânica, segundo Sartori et al. (2006) é a falta de conhecimento científico para o cultivo, pois a maioria das informações enfoca o cultivo orgânico em geral, de hortaliças, soja e café.
Em cultivo orgânico de tangerina, na Espanha, foi observada maior micoflora ambiental do que em pomares convencionais (Palou et al., 2001a), possivelmente relacionada à intensificação da vida microbiológica do solo e ao aumento da biodiversidade relatada nos sistemas de cultivo orgânicos (França et al., 2007).

O presente trabalho teve por objetivos: a) identificar e quantificar as doenças pós-colheita em laranjas 'Valência' provenientes de cultivo orgânico e convencional; b) identificar e quantificar a micoflora ambiental nos pomares citrícolas e c) detectar a presença de isolados de Penicillium spp. resistentes aos fungicidas tiabendazol e imazalil nos pomares.

\section{MATERIAL E MÉTODOS}

As amostragens dos frutos de laranja 'Valência' e da micoflora ambiental foram realizadas mensalmente, de julho a dezembro de 2008 e 2009 , em dois pomares orgânicos e em dois pomares convencionais, localizados em Borborema (latitude $-21^{\circ} 37^{\prime} 11^{\prime \prime}$ e longitude $-49^{\circ} 04^{\prime} 25^{\prime \prime}$ ) e Itápolis, SP (latitude $-21^{\circ} 35^{\prime} 44^{\prime \prime}$ e longitude $-48^{\circ} 48^{\prime}$ 46 "). Amostragens de frutos de laranja 'Valência' orgânicos comercializados na Companhia de Armazéns Gerais de São Paulo (CEAGESP) foram realizadas mensalmente no respectivo período, com frutos procedentes de pelo menos quatro municípios: Borborema, SP (três amostras), Domingos Martins, ES (três amostras), Gonçalves, MG (quatro amostras) e Cooperativa dos Citricultores Ecológicos do Vale do Caí, RS (Ecocitrus) (duas amostras). A incubação, identificação e quantificação das doenças pós-colheita e da micoflora ambiental foram realizadas nas dependências da APTA Centro Oeste Bauru, SP.

No manejo fitossanitário das doenças nos pomares orgânicos de Borborema e Itápolis, SP, com idades de oito e 18 anos, respectivamente, foram feitas, em média, pelos produtores 3,5 aplicações de calda sulfocálcica/ ano (2008 e 2009) e uma aplicação de calda bordalesa/ ano (2008); enquanto nos pomares convencionais de Borborema e Itápolis, SP, com idades de seis e 18 anos, respectivamente, foram feitas 5,8 aplicações de oxicloreto de cobre/ano (2008 e 2009), uma aplicação de piraclostrobina ou trifloxystrobina/ano (2008 e 2009) e uma aplicação de carbendazim/ano (2009).

\section{Identificação e quantificação das doenças pós-colheita}

Cem frutos de laranja 'Valência' foram coletados mensalmente e acondicionados em engradados plásticos higienizados em cada um dos quatro pomares amostrados, colhendo-se manualmente e aleatoriamente cinco frutos da porção mediana de 20 árvores. Os 100 frutos orgânicos amostrados em um atacadista na CEAGESP estavam sendo comercializados a granel e mantidos em engradados plásticos ou caixas de papelão.

Os frutos foram individualizados em bandejas plásticas e submetidos à câmara úmida por 24 horas, visando favorecer a expressão de sintomas de doenças, 
permanecendo por mais 13 dias a $25^{\circ} \mathrm{C}$ e $85 \%$ de umidade relativa, em câmara fria. Aincidência de doenças pós-colheita foi avaliada visualmente a cada dois-três dias. Em caso de dúvida na identificação da doença, efetuou-se o isolamento do fitopatógeno em meio batata-dextrose-ágar (BDA) e a patogenicidade testada através da inserção de discos de micélio em frutos sadios, previamente desinfestados superficialmente com $\mathrm{NaClO}(0,5 \%)$ e feridos, segundo metodologia de Nery-Silva et al. (2007). Os fungos isolados de frutos doentes tiveram a patogenicidade confirmada por meio da inoculação em frutos sadios.

\section{Identificação e quantificação da micoflora ambiental de pomares citrícolas}

A micoflora ambiental de cada pomar avaliado foi amostrada pelo método gravimétrico. Cinco placas de Petri de $9 \mathrm{~cm}$ de diâmetro (repetições) com meio BDA suplementado com $0,4 \mathrm{~g} \mathrm{~L}^{-1}$ de pentabiótico veterinário $\left(\right.$ Fort Dodge $\left.^{\circledR}\right)$ foram distribuídas entre duas linhas contínuas de árvores e abertas por um minuto para a deposição por gravidade de esporos fúngicos sobre o meio de cultivo. As amostragens foram realizadas sempre entre as 10 e 12 horas, como forma de minimizar a heterogeneidade das condições ambientais (Palou et al., 2001a).

As placas de Petri foram mantidas a $25^{\circ} \mathrm{C}$ e fotoperíodo de $12 \mathrm{~h}$, durante cinco a sete dias, com posterior contagem e identificação das colônias fúngicas. A identificação foi feita por gênero, exceto para as espécies $P$. digitatum e $P$. italicum, identificadas visualmente pela coloração da colônia e patogenicidade em frutos cítricos, e quando necessário, realizou-se observação microscópica (Barnett \& Hunter, 1998). A frequência de cada gênero fúngico detectado foi expressa como número de unidades formadoras de colônias (ufc) por placa.

\section{Determinação da frequência de isolados de Penicillium spp. resistentes a fungicidas}

A presença de isolados de Penicillium spp. resistentes aos fungicidas tiabendazol (Tecto SC) e imazalil (Magnate $500 \mathrm{CE}$ ) foi determinada nos pomares avaliados concomitantemente à amostragem da micoflora. Para a tomada de amostras, placas de Petri foram preparadas com meio de cultura BDA acrescido de $0,4 \mathrm{~g} \mathrm{~L}^{-1}$ de pentabiótico veterinário e de diferentes fungicidas e dosagens: (1) Sem fungicida; (2) $10 \mathrm{mg} \mathrm{L}^{-1}$ de ingrediente ativo (i.a.) de tiabendazol; (3) $1 \mathrm{mg} \mathrm{L}^{-1}$ de i.a. de imazalil; e (4) 10 $\mathrm{mg} \mathrm{L}^{-1}$ de i.a. de tiabendazol $+1 \mathrm{mg} \mathrm{L}^{-1}$ de i.a. de imazalil. Estas doses de fungicidas correspondem às chamadas doses de resistência, obtidas experimentalmente, e nas quais se considera que unicamente os isolados resistentes são capazes de crescer (Brown, 1990; Holmes \& Eckert, 1999; Zhu et al., 2006). O tiabendazol e o imazalil, assim como o pentabiótico veterinário, foram adicionados ao meio de cultura BDA fundente. A amostragem e incubação das placas foram realizadas da mesma forma descrita no item anterior, entretanto, para o ensaio de resistência, utilizaram- se três placas de Petri de cada meio de cultura, as quais permaneceram abertas por dois minutos nos pomares. No processo de contagem foram diferenciados três grupos, identificando as colônias visualmente pela coloração e por patogenicidade em frutos cítricos: $P$. digitatum, $P$. italicum e outras espécies de Penicillium.

Frutos com sintomas de bolor verde ou azul, coletados nos pomares e na CEAGESP, tiveram os conídios de $P$. digitatum ou $P$. italicum, respectivamente, repicados, com auxílio de uma agulha histológica, da superfície de frutos doentes para placas de Petri contendo os quatro meios de cultura acima descritos, visando determinar ou não a resistência do patógeno aos fungicidas através do seu crescimento micelial. Para cada fruto doente e meio de cultura foram empregadas três placas de Petri (repetições).

\section{Análise dos dados}

Os dados de incidência das doenças pós-colheita nos pomares e entre os pomares foram transformados em $(\mathrm{x}+0,5)^{1 / 2}$ e submetidos a uma análise de variância fatorial (4 pomares $\mathrm{x} 9$ doenças) e comparação de médias pelo teste de Tukey $(P<0,05)$, tomando-se as médias mensais (2008 e 2009) como repetições. Optou-se por analisar os pomares isoladamente em função da variabilidade entre os pomares de mesmo sistema de cultivo, considerando as diferentes idades e localidades.

As frequências dos gêneros fúngicos mais comuns e de isolados de Penicillium spp. resistentes a fungicidas foram transformadas em $(\mathrm{x}+0,5)^{1 / 2} \mathrm{e}$ as médias comparadas pelo teste de Tukey $(P<0,05)$ em relação aos diferentes pomares, tomando-se as médias de cada amostragem como repetição. A comparação entre amostragens da microflora nas diferentes avaliações de cada pomar foi feita com a mesma transformação, tomando-se as placas como repetições. A correlação entre a incidência de bolor verde nos frutos coletados nos pomares e a frequência de $P$. digitatum da micoflora ambiental local foi feita pela correlação linear (coeficiente de Pearson), a 5\% de probabilidade. Análise de correlação também foi realizada entre a micoflora total nos pomares e os dados climáticos de temperatura ambiente dos pomares e precipitação acumulada no município de Itápolis, SP (pluviômetro localizado a $480 \mathrm{~m}$ de altitude, latitude $21^{\circ} 35^{\prime}$, longitude $48^{\circ} 49^{\prime}$ ).

\section{RESULTADOS E DISCUSSÃO}

\section{Doenças pós-colheita}

A incidência total de doenças pós-colheita em laranja 'Valência' diferiu estatisticamente entre os quatro pomares amostrados aos 14 dias de armazenamento, com maiores incidências nos frutos do sistema orgânico em relação aos do sistema convencional (Tabela 1). Maiores incidências da podridão peduncular de lasiodiplodia foram observadas nos frutos orgânicos (14,8-20,3\%), assim como da podridão peduncular de phomopsis (Phomopsis citri Fawcettt) nos frutos orgânicos do pomar de Borborema (4,3\%). Na média 
Doenças pós-colheita em laranja 'Valência' e caracterização da população fúngica em pomares...

TABELA 1 - Incidência (\%) de doenças pós-colheita em laranjas 'Valência' produzidas em sistema orgânico (O) e convencional (C), aos 14 dias de armazenamento a $25^{\circ} \mathrm{C}$

\begin{tabular}{|c|c|c|c|c|c|}
\hline \multirow[t]{2}{*}{ Doenças } & \multicolumn{5}{|c|}{ Procedências $^{2}$ - Sistema de cultivo } \\
\hline & IC & $\mathbf{B C}$ & IO & BO & Média \\
\hline Podridão de lasiodiplodia & $6,0 \mathrm{cA}^{3}$ & $0,3 \mathrm{dA}$ & $14,8 \mathrm{bA}$ & $20,3 \mathrm{aA}$ & $10,4 \mathrm{~A}$ \\
\hline Podridão de phomopsis & $2,2 \mathrm{bB}$ & $0,2 \mathrm{bA}$ & $3,1 \mathrm{bB}$ & $4,3 \mathrm{aB}$ & $2,4 \mathrm{~B}$ \\
\hline Bolor verde & $1,4 \mathrm{aBC}$ & $0,9 \mathrm{aA}$ & $0,6 \mathrm{aC}$ & $0,4 \mathrm{aC}$ & $0,8 \mathrm{C}$ \\
\hline Antracnose & $0,1 \mathrm{aC}$ & $0,3 \mathrm{aA}$ & $0,1 \mathrm{aC}$ & $0,3 \mathrm{aC}$ & $0,2 \mathrm{C}$ \\
\hline Podridão negra & $0,1 \mathrm{aC}$ & $0,1 \mathrm{aA}$ & $0,1 \mathrm{aC}$ & $0,4 \mathrm{aC}$ & $0,2 \mathrm{C}$ \\
\hline Podridão de fusarium & $0,1 \mathrm{aC}$ & $0,4 \mathrm{aA}$ & $0,0 \mathrm{aC}$ & $0,3 \mathrm{aC}$ & $0,2 \mathrm{C}$ \\
\hline Podridão azeda & $0,2 \mathrm{aC}$ & $0,1 \mathrm{aA}$ & $0,0 \mathrm{aC}$ & $0,0 \mathrm{aC}$ & $0,1 \mathrm{C}$ \\
\hline Bolor azul & $0,1 \mathrm{aC}$ & $0,0 \mathrm{aA}$ & $0,2 \mathrm{aC}$ & $0,0 \mathrm{aC}$ & $0,1 \mathrm{C}$ \\
\hline Podridão de aspergilus & $0,0 \mathrm{aC}$ & $0,1 \mathrm{aA}$ & $0,0 \mathrm{aC}$ & $0,1 \mathrm{aC}$ & $0,0 \mathrm{C}$ \\
\hline Total & $10,1 \mathrm{c}$ & $2,4 \mathrm{~d}$ & $18,8 \mathrm{~b}$ & $25,9 \mathrm{a}$ & 14,3 \\
\hline
\end{tabular}

${ }^{1}$ Média de 12 amostragens mensais de 100 frutos (julho a dezembro de 2008 e 2009)

${ }^{2}$ Borborema $=(\mathrm{B})$ e Itápolis $=(\mathrm{I})$.

${ }^{3}$ Valores seguidos pela mesma letra, minúscula na linha e maiúscula na coluna, não diferem entre si pelo teste de Tukey, em nível de 5\%.

dos pomares, a podridão de lasiodiplodia foi a doença mais frequente $(10,4 \%)$, seguida da podridão peduncular de phomopsis $(2,4 \%)$. Bolor verde, antracnose, podridão negra (Alternaria citri Ellis \& N. Pierce), podridão de fusarium (Fusarium spp.), podridão azeda [Geotrichum citriaurantii (Ferraris) E. E. Butler], bolor azul (P. italicum) e podridão de aspergilus (Aspergillus niger Tieghem) foram encontradas em menor frequência $(<1,5 \%)$, não havendo diferenças entre si e entre os quatro pomares amostrados.

Maior incidência de doenças pós-colheita foi observada no pomar orgânico de Borborema já a partir do sexto dia de armazenamento $(2,7 \%)$, enquanto o pomar orgânico de Itápolis diferiu do pomar convencional de Itápolis a partir do $12^{\circ}$ dia (Figura 1). Menor incidência de doenças foi observada no pomar convencional de Borborema, com menos de $1 \%$ aos nove dias de armazenamento.

Diferenças significativas na incidência total de doenças pós-colheita nos pomares foram observadas entre os meses amostrados. De maneira geral, observouse um acréscimo na incidência total de doenças nos meses de novembro e dezembro em relação aos meses iniciais de amostragem (julho e agosto) (Figura 2).

Nos frutos de laranja 'Valência' orgânica, comercializados na CEAGESP, a incidência total de podridões foi de $26,9 \%$, próximo da incidência observada em laranja orgânica do pomar de Borborema $(25,9 \%)$ (Tabela 1). A principal doença detectada foi a podridão peduncular de lasiodiplodia $(11,9 \%)$, seguida pelo bolor verde $(7,2 \%)$ e pela podridão peduncular de phomopsis $(5,8 \%)$. Em menores incidências foram observados o bolor azul $(1,3 \%)$, a podridão de fusarium $(0,4 \%)$, a podridão negra $(0,3 \%)$ e a antracnose $(0,2 \%)$.

Os resultados obtidos nos pomares e na CEAGESP confirmam a importância econômica das podridões pós-colheita em citros, uma vez que essas doenças desqualificam a fruta para comercialização, como destacado por Eckert (1993), que mencionou perdas de até $50 \%$ durante o período de comercialização. A incidência comparativamente inferior de podridões nos frutos de laranja 'Valência' produzidas no sistema convencional em relação à orgânica deve-se, provavelmente, ao manejo fitossanitário mais intensivo nos pomares convencionais com fungicidas cúpricos e uso de fungicidas de ação mesostêmica/sistêmica mais específicos. Aplicações de tiofanato metílico em pré-colheita reduziram pela metade a incidência de podridões em frutos cítricos na Flórida, após o armazenamento (Sarvatore \& Ritenour, 2007). É importante salientar que há poucas opções de insumos certificados na agricultura orgânica para o controle de pragas e doenças quando comparado com a agricultura convencional. Segundo Turra (2005) as principais dificuldades de produção encontradas pelos produtores de laranja orgânica são o controle de pragas e doenças e a disponibilidade de mão de obra especializada.

Diferenças na incidência das podridões entre os períodos de coleta de frutos, nos pomares e na CEAGESP, também foram verificadas em amostragens em packinghouses e centrais atacadistas por Dantas et al. (2003) e Fischer et al. (2007, 2008), e, provavelmente, estão associadas à procedência dos frutos e as condições ambientais em cada mês de amostragem, pois a frequência e a intensidade das doenças são fortemente influenciadas pela variação ambiental imposta durante todas as etapas da pré e da pós-colheita (Agrios, 2005).

A podridão peduncular de lasiodiplodia foi a doença mais frequente em laranjas 'Valência' amostradas nos pomares e na CEAGESP, corroborando os resultados obtidos por Fischer et al. (2009b) com frutos da mesma variedade, destinados ao mercado externo. A doença assume maior importancia em regiões quentes e úmidas, como Flórida e Caribe (Brown \& Eckert, 2000). A elevada incidência desta doença em laranja 'Pêra' 


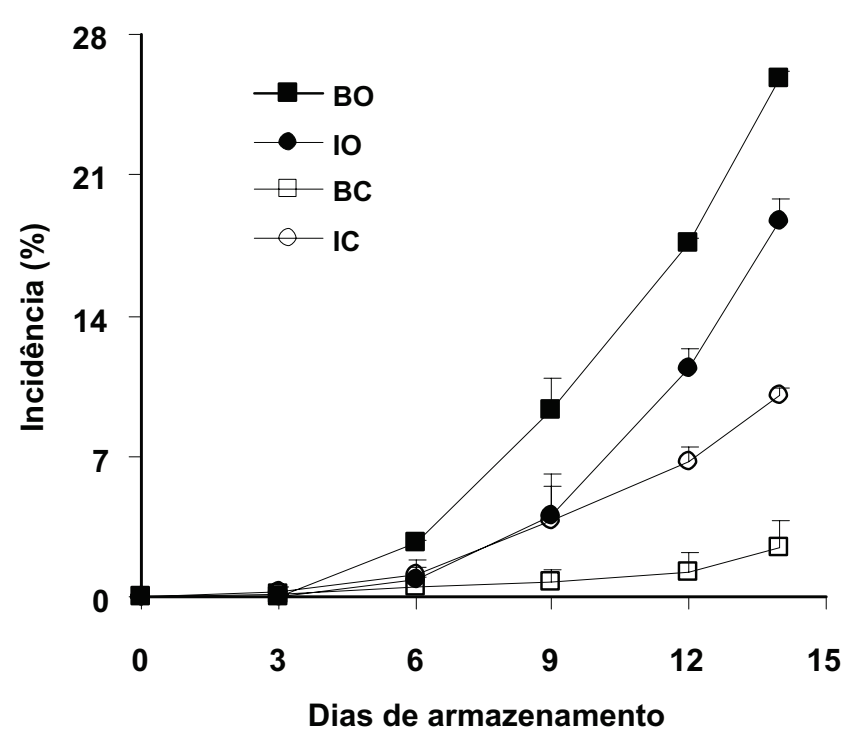

FIGURA 1 - Progresso da incidência total (\%) de doenças póscolheita em laranjas 'Valência' produzidas em sistema orgânico (O) e convencional (C), nas cidades de Borborema (B) e Itápolis (I), no estado de São Paulo, durante 14 dias de armazenamento a $25^{\circ} \mathrm{C}$. As barras representam o erro padrão da média de 2008 e 2009.

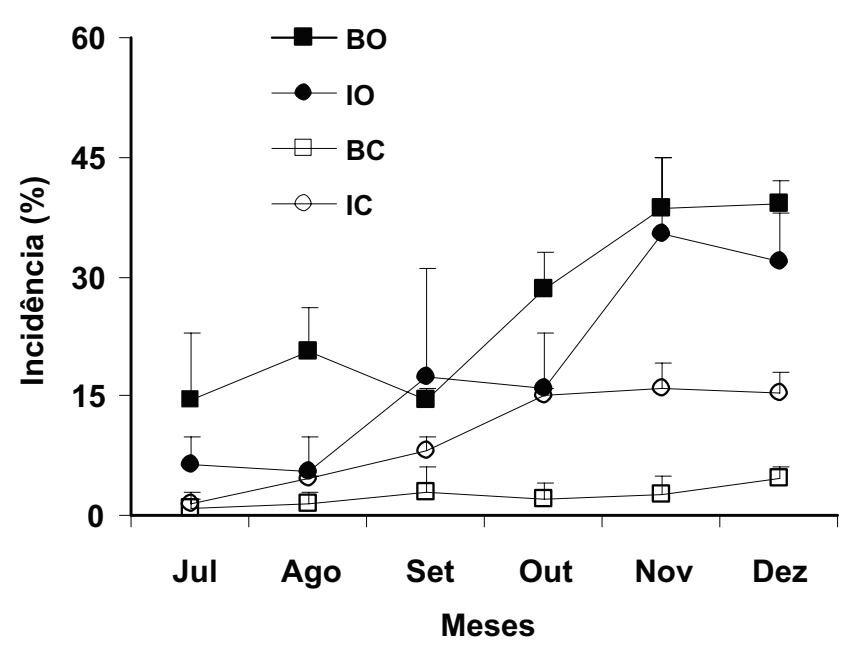

FIGURA 2 - Incidência mensal (\%) de doenças pós-colheita em laranjas 'Valência' produzidas em sistema orgânico $(\mathrm{O})$ e convencional (C), nas cidades de Borborema (B) e Itápolis (I), no estado de São Paulo. As barras representam o erro padrão da média de 2008 e 2009.

(11,8\%), em Recife, foi atribuída à temperatura do local de armazenamento dos frutos, em torno de $30^{\circ} \mathrm{C}$, aliada ao uso do etileno para o desverdecimento dos frutos (Dantas et al., 2003), que causa a abscisão precoce do cálice, facilitando a entrada do patógeno que se encontra latente nesse tecido (Barmore \& Brown, 1985).
Phomopsis citri se desenvolve em direção à região central do fruto, principalmente pela columela, semelhante a $L$. theobromae, porém mais lentamente e, raramente, atinge a extremidade estilar, causando perdas de maiores proporções somente quando os frutos demoram a ser comercializados (Ismail \& Zhang, 2004). Aumento na incidência dessa doença foi observado por Fischer et al. (2007), principalmente a partir da segunda semana de armazenamento em laranjas 'Lima' e 'Natal'. A importância de $P$. citri também foi verificada na Austrália e no Japão (Singh et al., 1977). O fungo que provoca a doença é o mesmo que provoca a melanose (Diaporthe citri) no período pós-florada, outra doença de citros.

$\mathrm{O}$ bolor verde, considerado a principal doença pós-colheita de laranjas e tangerinas (Citrus reticulata) em regiões semiáridas, podendo em adição ao bolor azul, representar mais de $90 \%$ do total de danos, durante as fases de transporte, armazenamento e comercialização (Tuset, 1987; Eckert \& Eaks, 1989; Agrios, 2005), foi pouco expressiva em laranja 'Valência', a exemplo do observado por Fischer et al. (2009b), com incidência média inferior a $1 \%$. Os mesmos autores, em estudo semelhante, verificaram que em laranjas 'Pêra, 'Natal' e 'Lima', produzidas e comercializadas no estado de São Paulo, o bolor verde foi a principal doença, com incidência superior a $10 \%$, sugerindo que a variedade 'Valência' possa ser menos suscetível. Outras doenças, como o bolor azul, a antracnose e a podridão azeda, embora em baixas incidências, podem ser importantes em determinadas condições de armazenamento (Ismail \& Zhang, 2004).

A grande diversidade de doenças pós-colheita provenientes de infecções latentes e/ou ativas sugere a necessidade do emprego de medidas de controle mais efetivas durante as fases de produção e pós-colheita. $\mathrm{O}$ uso de defensivos e as boas práticas agrícolas, incluindo adequada fertilização, podas de limpeza e remoção de frutos caídos no pomar, reduzem os tecidos vegetais mortos e consequentemente a fonte de inóculo de patógenos, como L. theobromae, P. citri, A. citri, C. gloeosporioides e Penicillium spp. Infecções em pós-colheita podem ser reduzidas por meio do manuseio cuidadoso dos frutos durante as operações de colheita, transporte, processamento e armazenamento; da utilização de drencher para a limpeza dos frutos antes de entrarem no packinghouse e da higienização, com produtos a base de cloro e amônia quaternária, das caixas de colheita e das instalações (máquinas, câmaras, piso, etc.) do packinghouse. Do mesmo modo, a redução das doenças pode ser obtida pelo emprego dos fungicidas em pós-colheita. Estima-se que sem o uso de tiabendazol em pós-colheita na Flórida, a venda de frutos cítricos poderia sofrer redução de até $50 \%$ (Ismail \& Zhang, 2004). A regulação do ambiente pelo uso de câmaras frias no armazenamento e contêineres refrigerados no transporte dos frutos beneficiados atrasa consideravelmente o desenvolvimento das doenças, sendo prática importante, especialmente se combinada com o tratamento químico, para a redução dos danos em pós-colheita. 
Identificação e quantificação da micoflora ambiental de pomares citrícolas

O número médio de colônias fúngicas por placa de Petri isoladas do ambiente dos pomares de laranja 'Valência', entre os anos de 2008 e 2009, foi de 28,7 (Tabela 2). Dentre as colônias fúngicas isoladas, 63,1\% corresponderam ao gênero Cladosporium, 9,3\% Fusarium, 7\% Epicoccum, 3,6\% Penicillium, sendo 1\% da espécie P. digitatum, 2,3\% Helminthosporium, 2,4\% Phitomyces, 2,1\% Alternaria, 0,7\% Aspergillus e os 9,4\% restantes aos gêneros Aureobasidium, Rhizopus, Trichoderma, Neurospora, Mucor, Cephalosporium, Cercospora, Colletotrichum, Curvularia, Fusicoccum, Geotrichum, Guignardia, Nigrospora, Pestalotia, Phoma, Phomopsis, Phytophthora, Septoria, Torula, Umicola, Tritirachium, Wallemia, Spegazzinia, Stachybotrys, Cryptosporium Polythrincium, Wardomyces entre outros. Observou-se apenas uma colônia de P. italicum, no pomar orgânico de Itápolis em 2008.

A micoflora total do ambiente, assim como a população dos principais gêneros fúngicos não diferiu significativamente entre os quatro pomares amostrados (Tabela 2). Entretanto, diferenças significativas $(P<0,05)$ na micoflora total de cada pomar foram observadas entre os meses amostrados (Figura 3). Palou et al. (2001a) encontraram maior micoflora ambiental em pomar orgânico de tangerina comparado aos pomares convencionais na Espanha. A micoflora total e a de Penicillium também apresentaram variabilidade elevada, com interações significativas entre épocas, packinghouses e pomares, havendo correlação positiva entre a micoflora total e a temperatura ambiente ( $r>0,9)$ (Palou et al., 2001a, 2001b). Na Turquia, maior micoflora ambiental foi observada no verão, seguida pelo outono, inverno e primavera (Simsekli et al, 1999).

Em 2009, concomitantemente às amostragens mensais da micoflora avaliou-se a temperatura do ar nos pomares. Não foram observadas correlações $(P<0,05)$ entre os dados de micoflora e temperatura nos quatro pomares, diferentemente da correlação positiva observada por Palou et al. (2001a).
Dados mensais de precipitação acumulada em Itápolis, SP, nos anos de 2008 e 2009, também não apresentaram correlação com a micoflora total amostrada mensalmente nos pomares de Itápolis, orgânico (IO) e convencional (IC) $(\mathrm{r}=-0,2$ em IC e r=-0,1 em IO), mesmo quando utilizada a precipitação acumulada nos últimos sete dias antes da amostragem da micofora ( $\mathrm{r}=0,1$ em IC e $\mathrm{r}=-0,2 \mathrm{em} \mathrm{IO})$.

Os resultados obtidos devem ser analisados levandose em consideração o método de amostragem utilizado para capturar esporos fúngicos dispersos pelo ar que, por sua vez, poderia explicar a ausência ou baixo isolamento de fungos dos gêneros Lasiodiplodia e Phomopsis, causadores das podridões pedunculares constatadas nos pomares (Tabela 1), mas que são disseminados basicamente por respingos de chuva (Brown \& Eckert, 2000). Diferenças de velocidade e características de crescimento entre os diferentes esporos coletados podem mascarar a presença de alguns gêneros fúngicos. $\mathrm{O}$ meio de cultivo empregado também pode favorecer o crescimento de alguns fungos em detrimento de outros (Palou et al., 2001b). Entre os patógenos pós-colheita com importância econômica, Penicillium e Alternaria foram os isolados com maior frequência, semelhante ao observado em pomares espanhóis (Palou et al., 2001a), packinghouses paulistas (Fischer et al., 2009a) e na CEAGESP (Fischer et al., 2008). Entretanto, a frequência de $P$. digitatum foi baixa quando comparada à encontrada em packinghouses paulistas, onde segundo Fischer et al. (2009a), eventualmente foram observados frutos podres sob a linha de beneficiamento, uma importante fonte de inóculo.

Embora a quantidade de inóculo fúngico influencie decisivamente na incidência de doenças, outros fatores como a suscetibilidade intrínseca dos frutos à infecção e as condições ambientais antes e durante o período de colheita também apresentam importância, impossibilitando o estabelecimento de uma relação quantitativa entre os níveis de população fúngica nos pomares e os danos por doenças pós-colheita(Palou etal.,2001a). Níveis de inóculo ambiental de $P$. digitatum e $P$. italicum em packinghouses citrícolas também não permitiram uma correlação significativa com os índices de bolores (Fischer, 2007). Entretanto, em ambiente dos atacadistas da CEAGESP, o mesmo autor observou

TABELA 2 - Colônias (unidades formadoras de colônias por placa) de gêneros fúngicos presentes no ambiente de pomares de laranja 'Valência' orgânicos e convencionais

\begin{tabular}{|c|c|c|c|c|c|c|c|c|c|c|c|}
\hline \multirow[t]{2}{*}{ Pomares $^{1}$} & \multicolumn{11}{|c|}{ População fúngica ${ }^{2}$} \\
\hline & Cladosporium & Fusarium & Epicoccum & Penicillium spp. ${ }^{3}$ & Helminthosporium & Pithomyces & Alternaria & P. digitatum & Aspergillus & Outros & Total \\
\hline $\mathrm{IC}$ & $22,6 a^{4}$ & $3,2 \mathrm{a}$ & $1,2 \mathrm{a}$ & $0,7 \mathrm{a}$ & $0,8 \mathrm{a}$ & $2,3 \mathrm{a}$ & $0,6 \mathrm{a}$ & $0,5 \mathrm{a}$ & $0,4 \mathrm{a}$ & $3,1 \mathrm{a}$ & $35,4 \mathrm{a}$ \\
\hline $\mathrm{BC}$ & $11,1 \mathrm{a}$ & $2,5 \mathrm{a}$ & $0,9 \mathrm{a}$ & $0,6 \mathrm{a}$ & $0,4 \mathrm{a}$ & $0,2 \mathrm{a}$ & $0,5 \mathrm{a}$ & $0,2 \mathrm{a}$ & $0,2 \mathrm{a}$ & $3,4 \mathrm{a}$ & $20,0 \mathrm{a}$ \\
\hline $\mathrm{IO}$ & $20,4 \mathrm{a}$ & $2,3 \mathrm{a}$ & $3,8 \mathrm{a}$ & $1,1 \mathrm{a}$ & $0,6 \mathrm{a}$ & $0,0 \mathrm{a}$ & $0,9 \mathrm{a}$ & $0,2 \mathrm{a}$ & $0,1 \mathrm{a}$ & $1,8 \mathrm{a}$ & $31,3 \mathrm{a}$ \\
\hline $\mathrm{BO}$ & $18,1 \mathrm{a}$ & $2,7 \mathrm{a}$ & $2,1 \mathrm{a}$ & $0,6 \mathrm{a}$ & $0,8 \mathrm{a}$ & $0,3 \mathrm{a}$ & $0,5 \mathrm{a}$ & $0,3 \mathrm{a}$ & $0,1 \mathrm{a}$ & $2,5 \mathrm{a}$ & $28,0 \mathrm{a}$ \\
\hline Média & 18,1 & 2,7 & 2,0 & 0,8 & 0,7 & 0,7 & 0,6 & 0,3 & 0,2 & 2,7 & 28,7 \\
\hline
\end{tabular}

${ }^{1} \mathrm{IC}=$ Itápolis convencional; $\mathrm{BC}=$ Borborema convencional; $\mathrm{IO}=\mathrm{Itáp}$ olis orgânico; $\mathrm{BO}=$ Borborema orgânico

${ }^{2}$ Médias de 14 amostragens mensais (junho a dezembro de 2008 e 2009).

${ }^{3}$ Espécies de Penicillium spp., exceto P. digitatum.

${ }^{4}$ Médias seguidas pela mesma letra na coluna não diferem entre si (Tukey, $\left.P<0,05\right)$. Análise estatística com os dados transformados $(\mathrm{x}+0,5)^{1 / 2}$ 


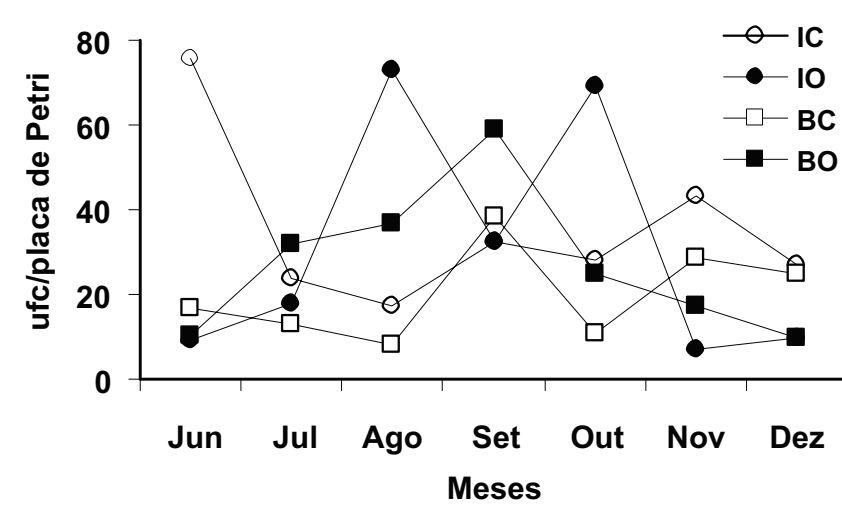

FIGURA 3 - População fúngica total isolada mensalmente (média entre 2008 e 2009) de ambiente de pomares de laranja 'Valência' com sistema de cultivo orgânico $(\mathrm{O})$ e convencional (C), localizados em Borborema (B) e Itápolis (I), SP.

correlação positiva entre a frequência de $P$. digitatum e o bolor verde em frutos de laranja 'Pêra' ali comercializados. Já para laranja 'Lima' e tangor 'Murcott' tal correlação não foi verificada (Fischer et al., 2008). No presente estudo, não foi observada correlação $(\mathrm{r}=0,7)$ entre a frequência de $P$. digitatum encontrada nos pomares paulistas e a incidência do bolor verde em frutos coletados nos respectivos pomares. Não existe um critério geral que permita discernir quais os limites críticos de contaminação fúngica a partir dos quais existe um alto risco de ocorrer uma incidência de bolor inadmissível. Não obstante, em packinghouses, procura-se definir, mediante os níveis fúngicos populacionais, zonas sujas e zonas limpas e também estabelecer limites críticos que permitam determinar a eficácia das operações de higienização (Sus \& Viñas, 1990; Orihuel et al.,1996; Palou et al., 2001b).

O nível da população fúngica ambiental presente nos pomares paulistas foi inferior ao encontrado em pomares citrícolas espanhóis com média de 52,9 ufc por placa (Palou et al., 2001a). Entretanto, assim como na Espanha, a contaminação fúngica nos pomares foi superior, comparativamente, a encontrada em packinghouses citrícolas (Fischer et al., 2009a; Palou et al., 2001b).

Cladosporium foi o gênero mais abundante nos pomares paulistas (Tabela 2), resultados que corroboram os encontrados por Palou et al. (2001a; 2001b) em pomares e packinghouses espanhóis e por Fischer et al. (2009a) em packinghouses paulistas. A espécie Cladosporium herbarum é descrita como um patógeno pós-colheita de pouca importância em citros (Tuset, 1987). Sua presença predominante nas superfícies das câmaras de desverdecimento e refrigeração de packinghouses citrícolas valencianos foi constatada por Díaz e Vila (1987, 1988). Em função da enorme variabilidade de colônias de Cladosporium encontrada neste trabalho e da baixa importância do mesmo como patógeno em citros, optou-se por não caracterizar as espécies.

\section{Determinação da frequência de isolados de Penicillium spp. resistentes a fungicidas}

Não foram observados isolados de $P$. digitatum resistentes a tiabendazol + imazalil no ambiente dos pomares citrícolas (Tabela 3). Um único isolado de P. digitatum resistente a imazalil foi observado no ambiente dos pomares convencionais de Itápolis em 2008 e de Borborema em 2009, correspondendo a uma frequência média de 0,02 ufc por placa. Resistência dos isolados de $P$. digitatum a tiabendazol foi constatada em porcentagens de 34,48 a 64,62 nos pomares, não havendo diferenças significativas na frequência $(0,12-0,24$ ufc por placa) entre os pomares. Penicillium italicum foi constatado em baixa frequência nas amostragens, apenas 1 ufc nos pomares de Itápolis e Borborema orgânico, não sendo observados isolados resistentes aos fungicidas. Em ambiente de packinghouses citrícolas paulistas a frequência de isolados de $P$. digitatum resistentes a imazalil também foi baixa $(<0,05$ ufc por placa), não sendo constatados isolados resistentes a tiabendazol + imazalil (Fischer et al., 2009a), entretanto, isolados de $P$. digitatum resistentes a tiabendazol foram observados em maior frequência $(0,36-2,36$ ufc por placa) no ambiente de dois packinghouses, possivelmente associada a maior população do patógeno nestes packinghouses (1,37-4,55 ufc por placa) em relação aos pomares $(0,21-0,69$ ufc por placa), uma vez que a porcentagem média de resistência foi de 47,3 nos pomares e 38,9 nos packinghouses.

A porcentagem média de outros Penicillium spp. resistentes a tiabendazol, imazalil e tiabendazol + imazalil foi de 27,94; 43,04 e 19,22, respectivamente, no ambiente dos pomares, sendo a frequência média de 0,$14 ; 0,22$ e 0,10 ufc por placa, respectivamente. A frequência de isolados de Penicillium spp. resistentes aos fungicidas não diferiu entre os pomares amostrados (Tabela 3 ). Semelhante ao observado nos pomares, amostragens da micoflora em packinghouses citrícolas paulistas e espanhóis evidenciaram maiores frequências de Penicillium spp. resistentes a imazalil e a tiabendazol + imazalil, quando comparado a $P$. digitatum e P. italicum (Palou et al., 2001b; Fischer et al., 2009a). Díaz \& Vila (1989) descreveram isolados de $P$. variabile, $P$. steckii e $P$. velutinum, e em menor escala de $P$. digitatum e $P$. italicum, com resistência cruzada a imazalil e procloraz. Em contrapartida, uma maior resistência a tiabendazol foi observada nos isolados de $P$. digitatum, em relação às demais espécies do gênero, nos pomares e packinghouses paulistas (Fischer et al., 2009a), diferentemente do constatado em packinghouses espanhóis (Palou et al., 2001b).

Dos isolados de $P$. digitatum obtidos de laranjas 'Valência' amostradas nos pomares orgânicos e convencionais (Tabela 1), 67,1\% oriundos do sistema convencional e $61,3 \%$ do sistema orgânico se mostraram resistentes in vitro ao fungicida tiabendazol, não sendo observada resistência ao imazalil e tiabendazol + imazalil. Já para os isolados de $P$. digitatum obtidos de laranjas 'Valência' amostradas na CEAGESP, 23,7\% mostraram-se resistentes ao tiabendazol e 2,6\% resistentes a imazalil e a 
Doenças pós-colheita em laranja 'Valência' e caracterização da população fúngica em pomares...

TABELA 3 - Frequência de Penicillium spp. resistentes aos fungicidas tiabendazol (TBZ) e imazalil (IZ) em ambiente de pomares de laranja 'Valência' orgânicos e convencionais

\begin{tabular}{|c|c|c|c|c|c|c|c|}
\hline \multirow[t]{2}{*}{ Isolados de Penicillium } & \multirow{2}{*}{$\begin{array}{c}\text { Tratamento } \\
\text { (ambiente) }\end{array}$} & \multirow{2}{*}{$\begin{array}{l}\text { Frequência } \\
\text { (Placa Petri) }\end{array}$} & \multirow[b]{2}{*}{ IC } & \multicolumn{3}{|c|}{ Pomares $^{1}$} & \multirow[b]{2}{*}{ Média } \\
\hline & & & & BC & IO & BO & \\
\hline \multirow[t]{7}{*}{ Penicillium digitatum } & $\mathrm{BDA}$ & ufc/placa & $0,69 \mathrm{a}^{2}$ & $0,35 \mathrm{a}$ & $0,21 \mathrm{a}$ & $0,26 \mathrm{a}$ & 0,38 \\
\hline & $\mathrm{BDA}+\mathrm{TBZ}$ & ufc/placa & $0,24 \mathrm{a}$ & $0,12 \mathrm{a}$ & $0,12 \mathrm{a}$ & $0,17 \mathrm{a}$ & 0,16 \\
\hline & & $\%$ & 34,48 & 34,48 & 55,56 & 64,62 & 47,29 \\
\hline & $\mathrm{BDA}+\mathrm{IZ}$ & ufc/placa & $0,02 \mathrm{a}$ & $0,02 \mathrm{a}$ & $0,00 \mathrm{a}$ & $0,00 \mathrm{a}$ & 0,01 \\
\hline & & $\%$ & 3,45 & 6,90 & 0,00 & 0,00 & 2,59 \\
\hline & $\mathrm{BDA}+\mathrm{TBZ}+\mathrm{IZ}$ & ufc/placa & $0,00 \mathrm{a}$ & $0,00 \mathrm{a}$ & $0,00 \mathrm{a}$ & $0,00 \mathrm{a}$ & 0,00 \\
\hline & & $\%$ & 0,00 & 0,00 & 0,00 & 0,00 & 0,00 \\
\hline \multirow{7}{*}{ Outros Penicillium spp. } & BDA & ufc/placa & $0,42 \mathrm{a}$ & $0,60 \mathrm{a}$ & $0,43 \mathrm{a}$ & $0,64 \mathrm{a}$ & 0,52 \\
\hline & $\mathrm{BDA}+\mathrm{TBZ}$ & ufc/placa & $0,13 \mathrm{a}$ & $0,18 \mathrm{a}$ & $0,13 \mathrm{a}$ & $0,13 \mathrm{a}$ & 0,14 \\
\hline & & $\%$ & 31,43 & 30,00 & 30,56 & 19,75 & 27,94 \\
\hline & $\mathrm{BDA}+\mathrm{IZ}$ & ufc/placa & $0,21 \mathrm{a}$ & $0,25 \mathrm{a}$ & $0,18 \mathrm{a}$ & $0,24 \mathrm{a}$ & 0,22 \\
\hline & & $\%$ & 51,43 & 42,00 & 41,67 & 37,04 & 43,04 \\
\hline & $\mathrm{BDA}+\mathrm{TBZ}+\mathrm{IZ}$ & ufc/placa & $0,01 \mathrm{a}$ & $0,10 \mathrm{a}$ & $0,17 \mathrm{a}$ & $0,12 \mathrm{a}$ & 0,10 \\
\hline & & $\%$ & 2,86 & 16,00 & 38,89 & 19,14 & 19,22 \\
\hline
\end{tabular}

*Médias de 14 amostragens mensais (junho a dezembro de 2008 e 2009).

${ }^{1} \mathrm{IC}=$ Itápolis convencional; $\mathrm{BC}=$ Borborema convencional; $\mathrm{IO}=$ Itápolis orgânico; $\mathrm{BO}=$ Borborema orgânico.

${ }^{2}$ Médias seguidas pela mesma letra na linha não diferem entre si (Tukey, $\left.P<0,05\right)$. Análise estatística com os dados transformados $(\mathrm{x}+0,5)^{1 / 2}$.

tiabendazol + imazalil. Os isolados de P. italicum obtidos de frutos amostrados nos pomares não foram resistentes aos fungicidas tiabendazol e imazalil, enquanto para os isolados obtidos de frutos cítricos da CEAGESP, 50\% foram resistentes a tiabendazol, $25 \%$ a imazalil e $15 \%$ a tiabendazol + imazalil. A proporção de isolados de $P$. digitatum resistentes a tiabendazol e imazalil também foi elevada em frutos cítricos, no Marrocos, 37 e 19\%, respectivamente, enquanto para P. italicum foi de 21 e 2,5\%, respecivamente. Já a resistência para ambos fungicidas foi de $10 \%$ para $P$. digitatum e 1,5\% para $P$. italicum (Boubaker et al., 2009).

A baixa frequência de isolados resistentes ao imazalil deve-se, provavelmente, a não utilização do grupo dos imidazóis em pomares citrícolas, além de ser, esse grupo químico considerado de baixo a mediano risco no desenvolvimento de resistência por patógenos (Decker, 1985). A maior frequência encontrada para o tiabendazol deve-se, provavelmente, ao uso contínuo de benzimidazóis (tiofanato metílico e carbendazim), em pomares citrícolas paulistas, no controle da podridão floral (Colletotrichum acutatum) e da mancha preta dos citros (Guignardia citricarpa), e que apresentam modo de ação similar ao tiabendazol (Fischer et al., 2009a). Isolados de P. digitatum resistentes se tornaram comuns em pomares japoneses onde benzimidazóis foram empregados por vários anos em pré-colheita (Kuramoto, 1976). Entretanto, os pomares convencionais estudados receberam apenas uma aplicação de carbendazim em 2009, indicando uma relativa baixa pressão de seleção aos benzimidazóis no período. Isolados resistentes a tiabendazol também foram encontrados em pomares e packinghouses onde não se aplicaram quaisquer benzimidazóis (Harding, 1972; Kuramoto, 1976; Wild \&
Eckert, 1982; Fischer et al., 2009a), a exemplo dos pomares orgânicos de laranja 'Valência', indicando a possibilidade de uma elevada variabilidade genética (Schmidt et al., 2006). Segundo Brown (1990), em uma população natural de $P$. digitatum, esporos resistentes a tiabendazol são produzidos na razão de 1 a 10 para cada 100 milhões de esporos produzidos, potencialmente, em um único fruto com bolor verde. Mutações nos genes $\beta$-tubulina e CYP51 têm sido descritas como responsáveis pela resistência de Penicillium spp. a tiabendazol e a imazalil, respectivamente (Schmidt et al., 2006; Zhu et al., 2006).

A frequência encontrada de isolados de $P$. digitatum resistentes aos fungicidas, principalmente ao tiabendazol, é preocupante, pois populações resistentes poderiam proliferar facilmente e também desenvolver resistência múltipla. Segundo Fischer et al. (2009a), a eficácia no controle de bolor verde, causado por isolados resistentes a tiabendazol, foi menor comparada aos isolados não resistentes, quando inoculados em frutos previamente tratados com o fungicida em sua dosagem comercial (1000 $\left.\mathrm{mg} \mathrm{L}^{-1}\right)$. Ao menos nos próximos anos não se prevê uma diminuição da pressão de seleção existente já que presumivelmente estes fungicidas continuarão a ser empregados de forma continuada. A combinação de fungicidas com diferentes modos de ação previne ou atrasa o desenvolvimento de populações resistentes (Delp, 1988). Entretanto, Holmes e Eckert (1999) verificaram que a proporção de isolados de $P$. digitatum e $P$. italicum com resistência tripla aos fungicidas imazalil, tiabendazol e ortofenilfenol praticamente duplicou-se em seis anos em packinghouses californianos devido à utilização intensiva destes fungicidas, tanto sequencialmente como em combinação. Produtos com diferentes modos de ação, como azoxistrobina, fludioxonil e pirimetanil, devem remediar os 
problemas de populações de Penicillium spp. resistentes ao tiabendazol e imazalil (Kanetis et al., 2008). Contudo, em trabalho recente estes autores já constataram uma menor sensibilidade in vitro de isolados de $P$. digitatum aos fungicidas fludioxonil e pirimetanil, mesmo antes de serem empregados comercialmente em pós-colheita (Kanetis et al., 2010).

\section{AGRADECIMENTOS}

Os autores agradecem à Fundação de Amparo à Pesquisa do Estado de São Paulo - FAPESP pelo apoio financeiro concedido ao projeto de pesquisa (Proc. 2007/08519-4), do qual resultou este artigo.

\section{REFERÊNCIAS BIBLIOGRÁFICAS}

Agrios GN (2005) Plant pathology. $5^{\text {th }}$ Ed. Amsterdam. Elsevier Academic Press.

Barmore CR, Brown GE (1985) Influence of ethylene on increased susceptibility of oranges to Diplodia natalensis. Plant Disease 69:228-230.

Barnett HL, Hunter BB (1998) llustrated genera of imperfect fungi. $4^{\text {th }}$ Ed. New York. Macmillan Publishing Company.

Boubaker H, Saadi B, Boudyach EH, Benaoumar AA (2009) Sensitivity of Penicillium digitatum and P. italicum to imazalil and thiabendazole in Morocco. Plant Pathology Journal 8:152-158.

Brown GE (1975) Factors affecting postharvest development of Colletotrichum gloeosporioides in citrus fruits. Phytopathology 65:404-409.

Brown GE (1990) Quality control assessment methodology related to citrus decay control. Lake Alfred USA. Florida Citrus Research and Education Center.

Brown GE, Eckert JW (2000) Diplodia stem-end rot. In: Timmer LW, Garnsey SM, Graham JH. (Eds.) Compendium of citrus diseases. $2^{\text {nd }}$ Ed. Saint Paul USA. APS Press. pp. 43-44.

Bus VG, Bongers AJ, Risse LA (1991) Occurrence of Penicillium italicum resistant to benomyl, thiabendazole, and imazalil on citrus fruit from different geographic origins. Plant Disease 75:10981100 .

Camargo AMMP, Caser DV, Camargo Filho WP, Camargo FP, Voelho PJ (2006) Área cultivada com agricultura orgânica no estado de São Paulo, 2004. Informações Econômicas 36:33-62.

Dantas SAF, Oliveira SMA, Michereff SJ, Nascimento LC, Gurgel LMS, Pessoa WRLS (2003) Doenças fúngicas pós-colheita em mamões e laranjas comercializados na Central de Abastecimento do Recife. Fitopatologia Brasileira 28:528-533.

Decker J (1985) Strategies for avoiding resistance to fungicides. In: Jenkyn JE, Plumb RT (Eds.) Strategies for the control of cereal disease. Oxford UK. Blackwell. pp.123-133.

Delp C.J (1988) Resistance management strategies for benzimidazoles. In: Delp CJ (Ed.) Fungicide resistance in North America. Saint Paul USA. APS Press. pp. 41-43.

Díaz MA, Vila R (1987) Estudio de flora fúngica presente en cámaras frigoríficas de conservación de frutos cítricos.

\section{Alimentaria 183:77-82.}

Díaz MA, Vila R (1988) Evolución de la flora fúngica durante la desverdizacíon en almacenes españoles de comercilaizacíon de cítricos. II. Flora presente en las cámaras de desverdización. Revista de Agroquímica y Tecnología de Alimentos 28:501-508.

Díaz MA, Vila R (1989) Imazalil resistant Penicillium isolates from Spanish citrus packinghouses. Microbiologie Aliments Nutrition 7:191-192.

Eckert JW (1993) Post-harvest diseases of citrus fruits. Agriculture Outlook 54:225-232.

Eckert JW, Eaks IL (1989) Postharvest disorders and diseases of citrus fruits. In: Reuter W, Calavan EC, Carman GE (Eds.) The citrus industry, v. 5. Berkeley USA. University of California Press. pp. 179-260.

Fischer I H (2007) Doenças pós-colheita em citros e caracterização da população fúngica em packing houses e na Ceagesp-SP. Tese de Doutorado. Piracicaba SP. Universidade de São Paulo.

Fischer IH, Toffano L, Lourenço AS, Amorim L (2007) Caracterização dos danos pós-colheita em citros procedentes de “packing house". Fitopatologia Brasileira 32:304-310.

Fischer IH, Lourenço AS, Amorim L (2008) Doenças pós-colheita em citros e caracterização da população fúngica ambiental no mercado atacadista de São Paulo. Tropical Plant Pathology $33: 219-226$.

Fischer IH, Afonseca LS, Spósito MB, Amorim L (2009a) Characterization of the fungal population in citrus packing houses. European Journal of Plant Pathology 123:449-460.

Fischer IH, Ferreira MD, Spósito MB, Amorim L (2009b) Citrus postharvest diseases and injuries related to impact on packing lines. Scientia Agricola 66:210-217.

França SC, Costa SMGS, Dias AD (2007) Microbial activity and arbuscular mycorrhizal fungal diversity in conventional and organic citrus orchards. Biological Agriculture \& Horticulture 25:91-102.

Gullino ML (1994) Lotta biologica a funghi agenti de arciumi della frutta in post-raccolta. Informatore Fitopatologico 4:5-13.

Harding PR Jr (1972) Differential sensitivity to thiabendazole by strains of Penicillium italicum and P. digitatum. Plant Disease Reporter 56:256-260.

Holmes GJ, Eckert JW (1999) Sensitivity of Penicillium digitatum and $P$. italicum to postharvest citrus fungicides in California. Phytopathology 89:716-721.

Houck LG (1977) Problems of resistance to citrus fungicides. Proceedings of the International Society of Citriculture 1:263269.

Ismail M, Zhang J (2004) Post-harvest citrus disease control. Outlooks on Pest Management 15:29-35.

Kanetis L, Förster H, Adaskaveq JE (2008) Optimizing efficacy of new postharvest fungicides and evaluation of sanitizing agents for managing citrus green mold. Plant Disease 92:261-269.

Kanetis L, Förster H, Adaskaveq JE (2010) Determination of natural resistance frequencies in Penicillium digitatum using a new air-sampling method and characterization of fludioxonil- and pyrimethanil-resistant isolates. Phytopathology 100:738 -46.

Nery-Silva FA, Machado JC, Resende MLV, Lima LCO (2007) 
Metodologia de inoculação de fungos causadores da podridão peduncular em mamão. Ciência e Agrotecnologia 31:1374-1379.

Orihuel B, Canet JJ, Bertó R(1996)Límites críticos e contaminación fúngica de superficies en una central hortofrutícola. Fruticultura Professional 83:114-118.

Palou L, Usall J, Pons J, Viñas I. (2001a) Micoflora epifita de los frutos y ambiental en campos de mandarino 'Clemenules' en Tarragona. Revista Investigación Agraria: Production Protección Vegetal 16:257-272.

Palou L, Usall J, Pons J, Cerda MC, Viñas I (2001b) Micoflora em centrales citrícolas de Tarragona. Revista Investigación Agraria: Production Protección Vegetal 16:447-462.

Sartori IA, Panzenhagen NV, Soares DN, Soglio FK, Manzete F, Koller OC (2006) Sistemas de cultivo orgânico e convencional em laranjas 'Valência'. Revista Brasileira de Agroecologia 1:207211.

Sarvatore JJ, Ritenour MA (2007) Effectiveness of different fungicides applied preharvest at reducing postharvest decay of fresh Florida citrus. Proceedings of the Florida State Horticultural Society 120:281-284.

Schmidt LS, Ghosoph JM, Margosan DA, Smilanick JL (2006) Mutation at B-tubulin codon 200 indicated thiabendazole resistance in Penicillium digitatum collected from California citrus packinghouses. Plant Disease 90:765-770.

Simsekli Y, Gücin F, Asan A (1999) Isolation and identification of indoor airborne fungal contaminants of food production facilities and warehouses in Bursa, Turkey. Aerobiologia 15:225-231.

Singh G, Rippon LE, Gilbert WS (1977) 2,4-D residues in stored lemons from post-harvest treatments. Australian Journal of Experimental Agriculture and Animal Husbandry 17:167-170.

Sus V, Viñas I (1990) Efecto de la desinfeccion sobre la contaminacion fungica de camaras frigorificas de fruta de pepita: evaluacion de desinfectantes en el control del Penicillium expansum. Microbiologie Aliments Nutrition 8:95-102.

Turra C (2005)Elementos químicos como critérios de discriminação de citros orgâncios de convencionais. Dissertação de Mestrado. Piracicaba SP. Universidade de São Paulo.

Tuset JJ (1987) Podredumbres de los frutos cítricos. Valência. Generalitat Valenciana.

Wild BL, Eckert JW (1982) Synergy between a benzimidazolesensitive isolate and benzimidazole-resistant isolates of Penicillium digitatum. Phytopathology 72:1329-1332.

Zhu JW, Xie QY, Li HY (2006) Occorrence of imazalil-resistance biotype of Penicillium digitatum in China and the resistance molecular mechanism. Journal of Zhejiang University Science $7: 362-365$

TPP331 - Recebido 27 Maio 2011 - Aceito 29 Dezembro 2011

Editor de Seção: Renato B. Bassanezi 